\title{
Surgical repositioning of impacted permanent central incisor-A Novel method and case report
}

\author{
Dr.E.Ramakrishnan ${ }^{1}$, Dr.Ameera Ahamed Ismail ${ }^{2}$, Dr. Ershad Ahamed ${ }^{2}$, \\ Dr.Sinu Jayaprakash ${ }^{2}$ \\ ${ }^{I}$ (Professor Dpt Of Pediatric Dentistry, KMCT Dental College,Manassery, Kozhikode,Kerala,India) \\ ${ }^{2}$ (Postgraduate Student,Dpt Of Pediatric Dentistry, KMCT Dental College,Manassery,Kozhikode,India
}

\begin{abstract}
Non-eruption of permanent central incisor due to impacted position and presence of obstructing supernumerary tooth is often seen in children. The commonest treatment done for this is surgical exposure and applying traction to bring it in to the alignment. When the impaction is very unfavourable for this treatment the extraction and prosthetic replacements were done. Surgical repositioning is an alternative treatment in such cases. In this case report a successful surgical repositioning of a permanent central incisor is presented.
\end{abstract}

Keywords: Impacted incisors,Surgical repositioning,Unerupted incisors.

\section{Introduction}

Impacted permanent central incisor in a child presents a difficult situation for the clinician. According to the Dental aesthetic index of university of IOWA 1986, a missing /unerupted maxillary permanent incisor can have a major impact on dental and facial esthetics and were considered to be the most unattractive deviant occlusal trait in their study ${ }^{[1]}$.In a hospital population the prevelance of impacted permanent incisor has been reported as $2.6 \%$ by Dibiase DD $1969^{[2]}$. The most commonly employed treatment in these cases is surgical exposure and orthodontic movement of the tooth in to alignment. When the tooth is placed deep and its position is unfavourable, this treatment is not possible. Here we have employed a novel method of surgical repositioning of impacted tooth in to normal alignment. A case report with results after 9months is presented here. This method is easy and takes less time for completion of treatment.

\section{Case report}

A 9 year old male child reported to the pediatric dentistry clinic, with complaint of unerupted 21.His IOPA xray showed a supernumerary tooth obstructing eruption of 21. Surgical removal of supernumerary was done to allow the eruption of 21.After 2 months it was found that 21 was not erupting due to lack of space on eruption of 22(fig:1,2). So it was decided to create space for 21.A removable appliance with coffin spring was given and it was activated every 2 weeks and sufficient space was created (fig:4,5). Waited for 9 months and 21 was not erupting .So it was decided to do a surgical repositioning of 21.

\section{Pre-surgical procedure}

An upper impression was made and a stone cast was made. 21 was made in wax in its place on the cast and a cap splint was made extending from 13 to 23 ,in selfcure. Child was advised to take tetanus toxoide injection, since he had not taken it during the past 2 years. He was advised to take $250 \mathrm{mg}$ capsule of amoxicillin starting from the morning on the day of operation, for four days. The parent was advised, not to frighten the child by talking of "operation" to others, in front of the child.

\section{Surgical procedure}

Local anesthetic infiltration was given on labial and palatal side of the area extending from 13 to 23 (fig:6).A curtain flap was raised and 21 was luxated using periosteal elevators and was brought down using an upper root forceps (fig:7,8). The reduced enamel epithelial sac was removed using an artery forceps and Bp blade (Fig:9). The tooth was positioned in its normal position and the flaps were sutured (fig:10). The cap splint was tried to see that 21 was remaining in correct position (fig:11). Then the cap splint was cemented using a thin mix of zincoxide eugenol in 21 area and zinc phosphate cement in the posterior area of splint (fig:12). After one week splint and sutures were removed. Then the cap splint was recemented for one more week. At the end of two weeks time cap splint was removed and 21 was seen firm in its position. Child was instructed to take care not to bite with new teeth for few more weeks. After 10 months, electric pulp testing gave positive response . Clinically, there was no discolouration of tooth ,no sign of infection and no mobility (fig:13). The root apex was not closed at the time of surgical repositioning (fig:14). IOPA xray after 9 months showed that the root formation was completed (fig:15). 
After 9 months there was no discolouration,no mobility,no signs of infection and gingival contour and gingival sulcus depth were normal and healthy.

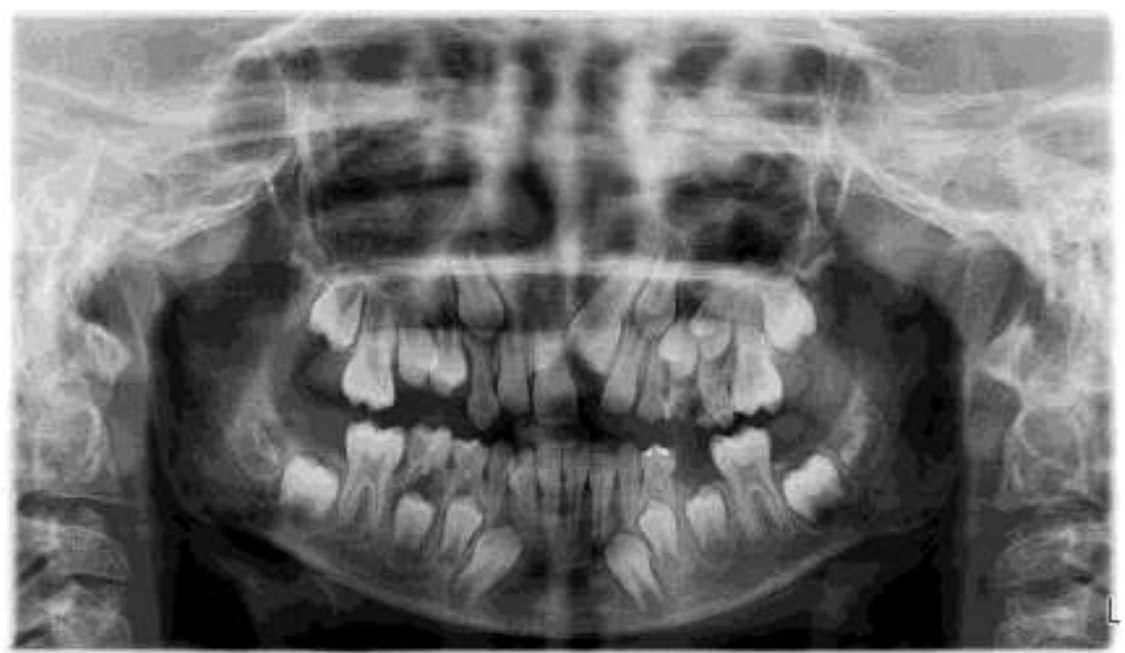

Fig1:-OPG showing impacted 21
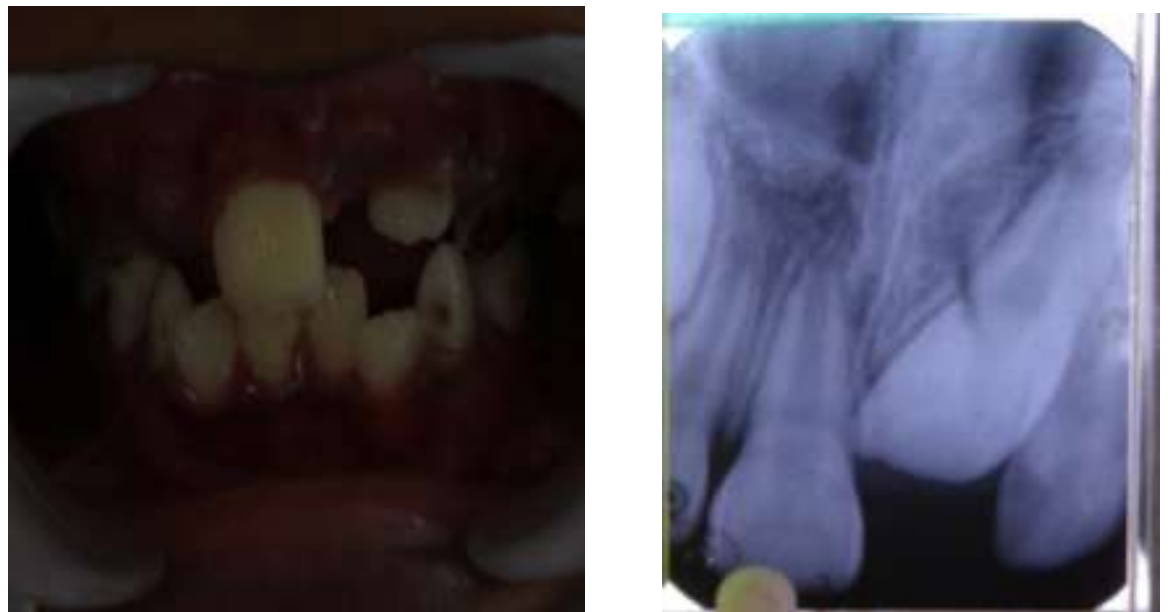

Fig:2- Pretreatment photograph showing

Fig:3- IOPA x ray showing impacted 2121 ,eruting 22 andlack of space for 11

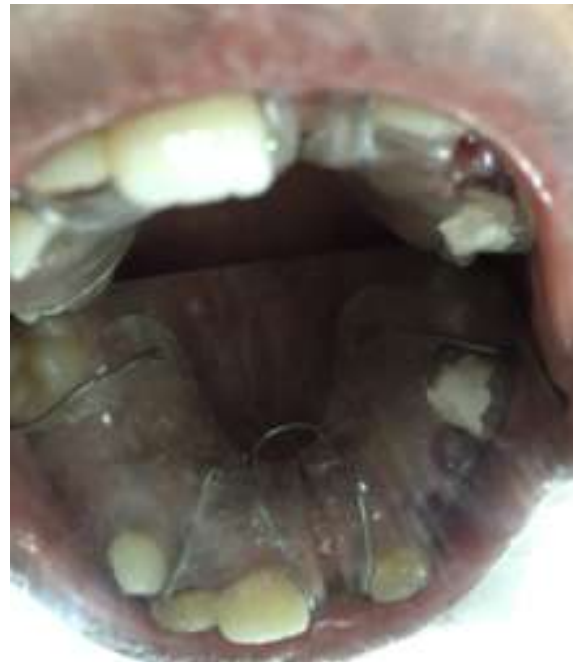

Fig4:-Mirror view of upper removable appliance to create space for 21

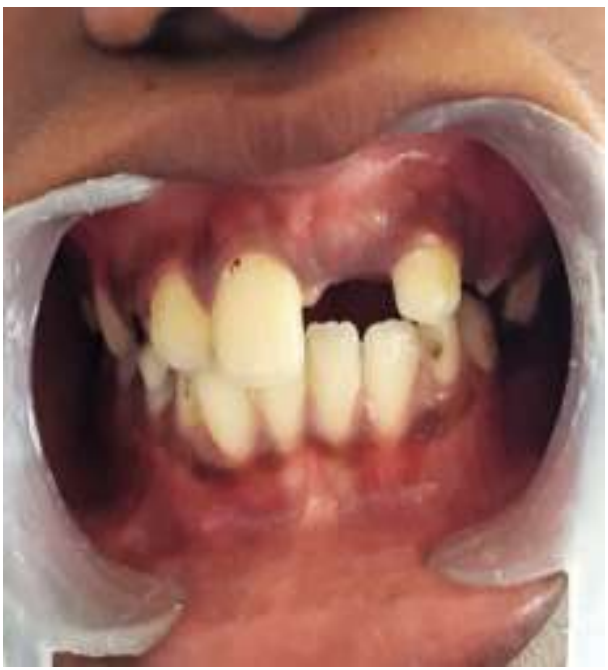

Fig5:-showing space created for 21 


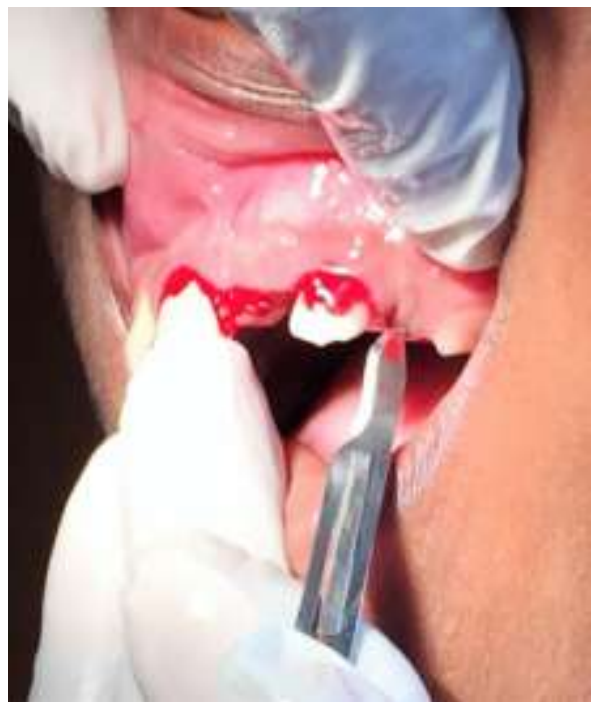

Fig6:-Incision for raising the labial flap

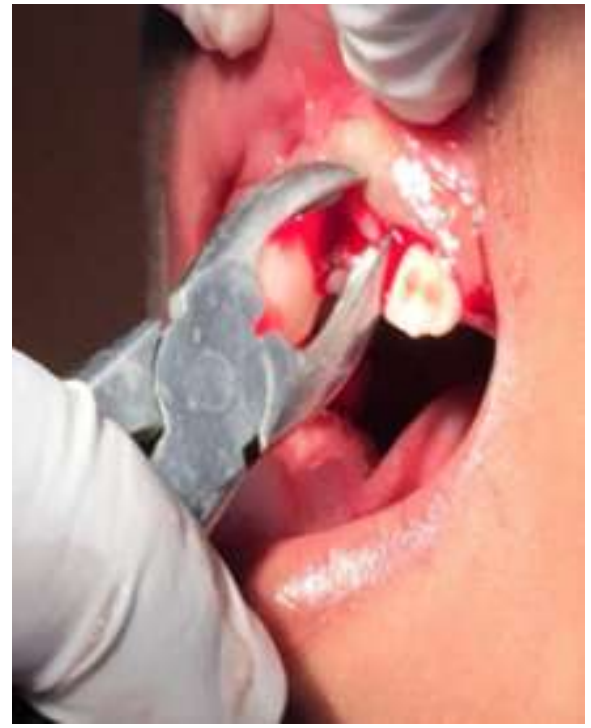

Fig8:-21 is brought down using forceps

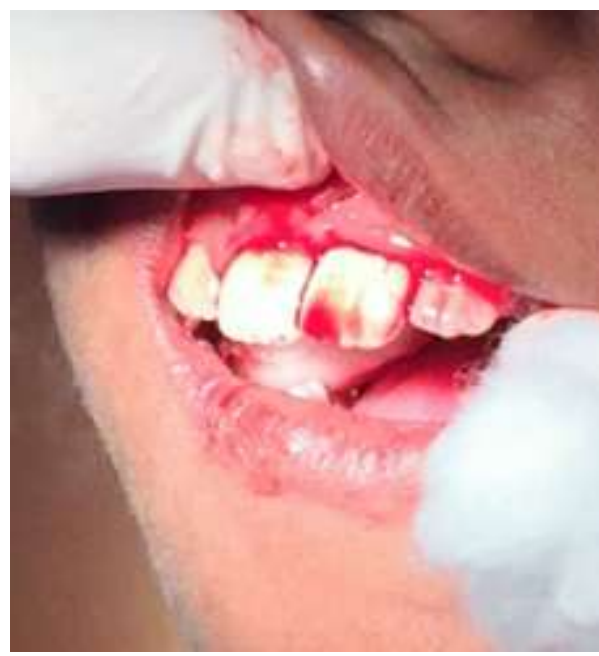

Fig10:-21 is brought in to alignment

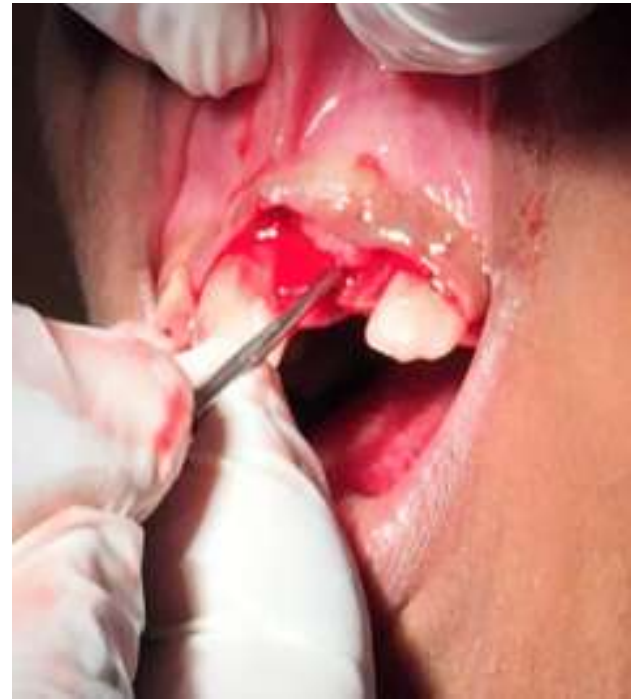

Fig7:-21 is luxated using periosteal elevator

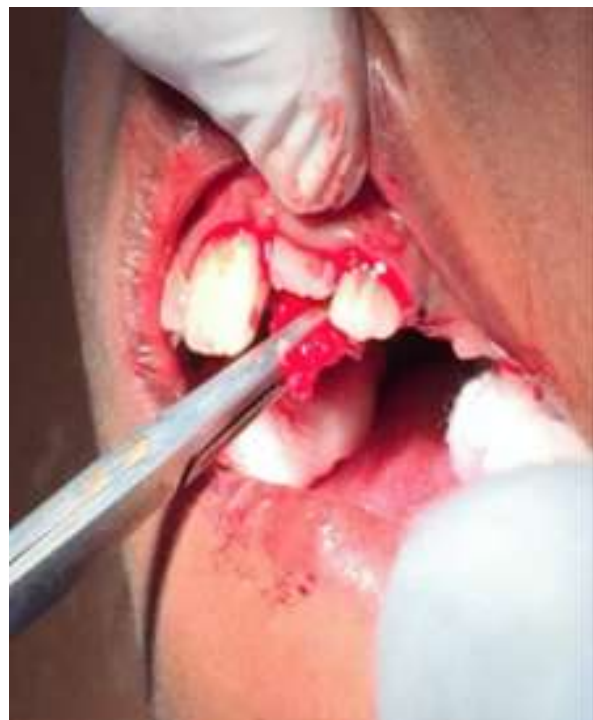

Fig9:-The enamel sac of 21 is detached and removed using artery forceps and blade

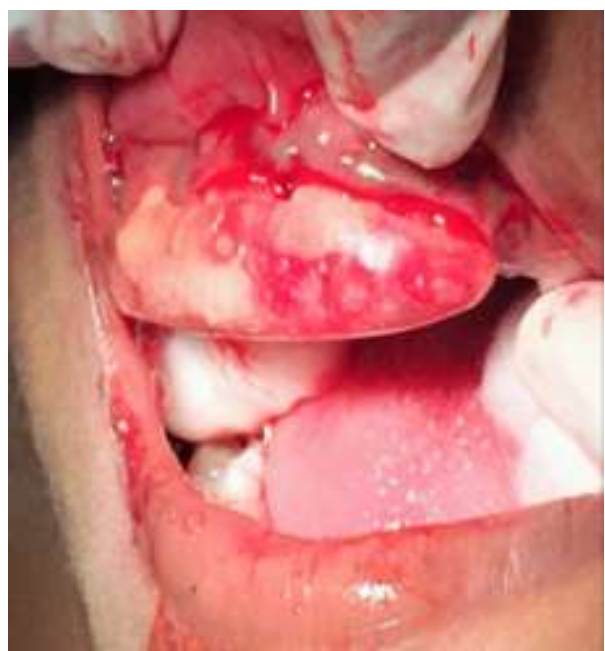

Fig11:-Cap splint is tried 


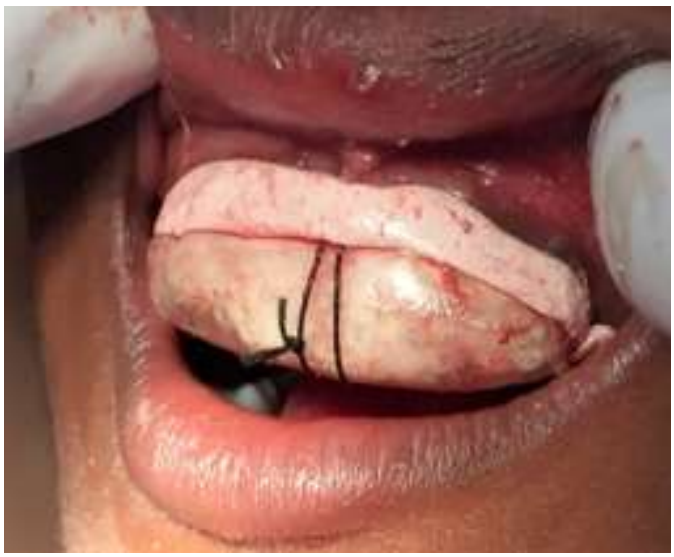

Fig12:-Cap splint is cemented Suture is placed \&ZOE pack is given

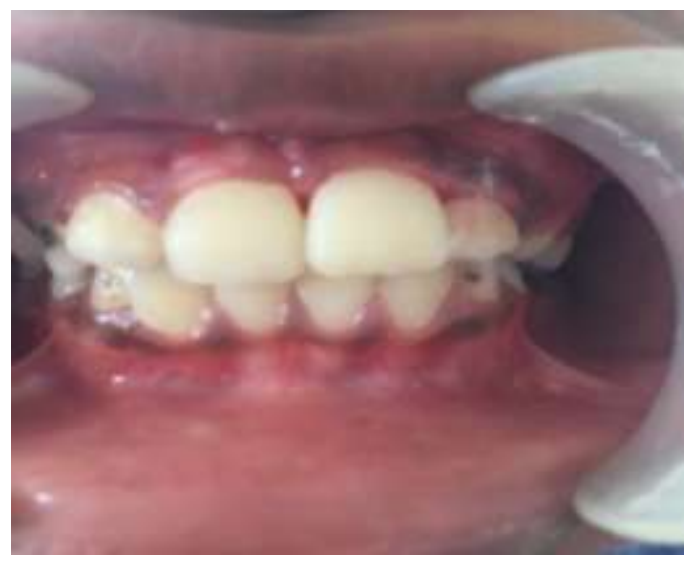

Fig13:-The repositioned 21,9 months after procedure

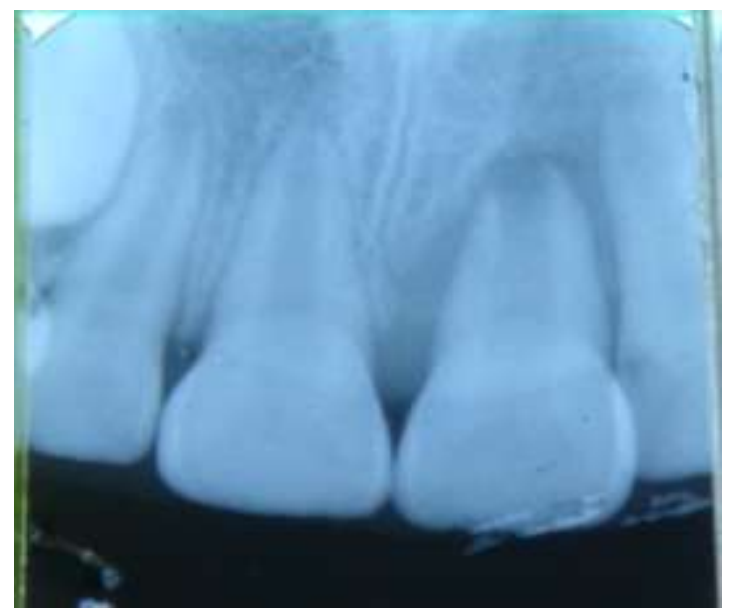

Fig 14:-IOPA xray taken one month after reimplantation. 21 with open apex

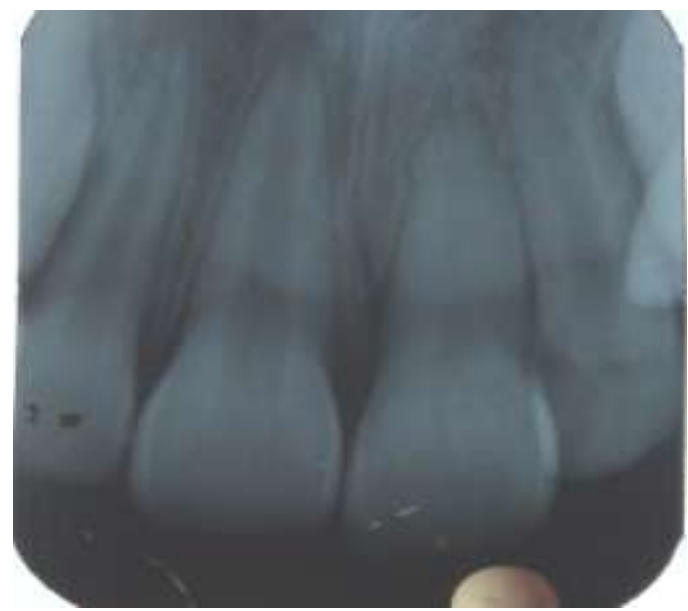

Fig 15:- IOPA xray after 9 months root apex is closed

\section{Discussion}

It is a challenging situation to the Pedodontist to treat an impacted permanent incisor in a child patient. To bring the natural tooth in to its proper position without loosing the vitality is the duty of the treating dentist. Many a times extractions and prosthetic replacements was done in such cases. The impacted and deeply placed tooth make it impossible for surgical exposure and application of traction to bring it in to alignment.The method of surgical repositioning is a newer,viable solution to this problem.Surgical repositioning is a kind of auto transplantation , as the tooth is completely moved from its impacted position to a new position at a distance of one or more centimeters. In this procedure the connection between the pulpal tissue in the rootcanal and periapical tissue is cutoff. So it is normally assumed that repositioned tooth loses its vitality and gets infected.Our experience in this procedure during the last 20 years is that the tooth when repositioned without any extra oral time,does not become discoloured or infected and has retained its vitality. We have observed this positive result not only in teeth with open apices, but also in teeth with closed apices. In this case report ,surgical repositioning of a tooth with open apex is presented.

Auto transplantation was first reported by Widman in $1915^{[3]}$. The transplantation is very successful when the root end is open. In this case root end was open at the time of replantation and the root end was found closed after a period of 9 months after repositioning.

Vivek kumar Adlakha $2011^{[4]}$ had reported good success in auto transplantation of dilacerated upper central incisor. Prabhu.N.T $1997^{[5]}$ has reported good successful surgical repositioning of permanent maxillary central incisor in a 12 year old boy. Andreason $1992^{[6]}$ states that if auto transplantation was carefully done success rate can be $95 \%$.

In our surgical repositioning (auto transplantation) procedure we had not done any endodontic treatment during or after the procedure. Here the extra oral time was nil. The tooth was moved from its impacted position and brought directly in to its new position, without taking it out of the surgical wound. Lena Baglund etal $1996^{[7]}$ recommends endodontic treatment in all closed apex cases. In our experience with closed apex 
cases,there were no indication for endodontic treatment post operatively. This may be because of the nil extra oral time in our procedure.

In this case we have used a cap splint for stabilizing the repositioned tooth for two weeks. Usually a flexible splint is preferred for stabilizing the replanted/repositioned tooth. In our cases the tooth is splinted with less rigidity by using thin mix of ZOE cement in the replanted tooth area. Also space in the splint in the repositioned tooth area is made larger in size, while fabricating it. The posterior part of cap splint is cemented with zinc phosphate cement for good retention of the splint. In our experience this splint serves the purpose of good stabilization.

The most commonly impacted maxillary tooth is canine followed by central incisor. The advantage of the surgical repositioning/auto transplantation is that it gives a quicker and satisfactory result in shorter period of time, as compared to the procedure of surgical exposure and use of traction to bring them in to alignment. When surgical exposure becomes impossible, due to deeper and abnormal position, extraction and prosthetic replacement were done. Surgical repositioning is definitly a good alternative in such cases. Autotransplantation done for immature tooth is always very successful. In this case the root end was open and after the reimplantation there was continued root end formation and apex closure.

\section{Conclusion}

Surgical repositioning procedure for an impacted maxillary central incisor with open apex is done with good results.The replanted tooth did not get discoloured or infected. It gave positive response to vitality tests.The gingival contour and gingival sulcus depth were normal. The root end was found closed at the end of 9 months post operatively. The results after 9 months is presented

\section{Declaration of patient consent}

The authors certify that they have obtained all appropriate patient consent forms.In the form the patient[s] has/have given his/her//their images and other clinical information to be reported in the journal. The patients understand that their names and initials will not be published and due efforts will be made to conceal their identity,but anonymity cannot be guaranteed.

\section{Financial support and sponsorship}

Nil.

\section{Conflicts of interest}

There are no conflicts of interest

\section{References}

[1]. Cons.n.c,Jenny.J,Khont F.J,DAI the dental aesthetic index.Iowa college of dentistry,university of lowa-1986

[2]. DiBiase D.D.Medline supernumeraries and eruption of maxillary central incisors Transaction of the BSSO 1968-1969;83-88

[3]. Widman .L.1915 om transplantation ab retrinerade horritander,svensk Tandlakartidskmift.

[4]. Vivek kumar Alakha ,Prutika CRandra,Sumir Gandhi,Saroj Chopra,Nmitha Singh,Shamsher singh Surgical repositioning of a Dilacerated impacted incisor:International Journal of Clinical Paediatric Dentistry,Jan-April-2011,4(1) 55-58

[5]. Prabhu.n.t,Munshi.A.K.,surgical management of a labially placed permanent maxillary central incisor after supernumerary teeth reimplantation:Report of a case.J.clinic.paediatric .Dent.1997 spring 21(3):201-3

[6]. Andreason J.O 1992.Auto Transplantation of canines .In Andreason J.O(ed).Atlas of replantation and transplantation of teeth.Chapter 6.Munksgaard .Cepenchagen PP-196.

[7]. Lena Berglund,Jon Curol and seven Koint orthodontic pretreatment prior to autotransplantation of palatally impacted maxillary canines.case report on a new approach.European Journal of OrthodonticS 18 (1996)449-456. 